\title{
Effect of lightness contrast on Ponzo illusions
}

\author{
TED JAEGER \\ Methodist College, Fayetteville, North Carolina 28301 \\ and \\ FRANK TREIBER and ROBERT H. POLLACK \\ University of Georgia, Athens, Georgia 30602
}

\begin{abstract}
Thirty-six undergraduates between 18 and 25 years of age judged the length of the enclosed line in tachistoscopically presented Ponzo illusion figures. Increasing the length of the sides of the Ponzo wedge created an overestimation of enclosed line length that was maximal with wedges of intermediate size. For all Ponzo figures studied, this illusion of overestimation was significantly affected by variations in the lightness contrast of the wedge and enclosed line. The data support the contention that the Ponzo and Muller-Lyer illusions are variants of each other and suggest that the former illusion must be at least partially attributable to a contour interaction process.
\end{abstract}

The existence of a similarity in the structural characteristics of the Ponzo and Muller-Lyer figures has been noted by Fisher (1967) and Pressey (1971). They point out that the juxtaposition of the enclosed line and the portion of the Ponzo wedge that extends outward from this line creates a pattern resembling an arrowfeather Muller-Lyer figure with half of each of its outward-going fins present. This figural similarity has led the aforementioned illusion theorists to argue that the inclined lines of the Ponzo and arrowfeather Muller-Lyer figures activate the same illusion-producing mechanism, thereby causing the enlargement of each figure's bounded line. Their claim seems substantiated both by Quina and Pollack's (1971) demonstration that apical lines in the Ponzo illusion figure are typically overestimated in length and by the findings of Day and Dickinson (1976) and Warren and Bashford (1977), which indicate that a reduced arrowfeather Muller-Lyer figure having only half of each fin present still produces overestimation of the enclosed line.

Despite Fisher's (1967) and Pressey's (1971) observation that portions of the Ponzo and Muller-Lyer figures bear a strong resemblance, it nonetheless remains the case that structural dissimilarities exist between the classical forms of these illusion figures. For example, in the classical Ponzo figure (illustrated in Figure 1), there is no intersection of the inclined wedge lines and the bounded line, whereas in the classical Muller-Lyer figure the inclined fin lines and bounded line do intersect. Also, in the classical Ponzo figure, the lines of the wedge meet, thus leaving the bounded line flanked on one side by inward-projecting line segments and on the other side

The data reported here were originally presented at the 1979 meeting of the Southern Society for Philosophy and Psychology, Norfolk, Virginia. Requests for reprints should be sent to Ted Jaeger, Department of Behavioral Sciences, Methodist College, Fayetteville, North Carolina 28301. by either inward- or outward-projecting lines, but not by combinations of each. Given these structural dissimilarities, it is uncertain whether Fisher's (1967) and Pressey's (1971) claim of a common visual process underlying both illusions could be valid only for the structurally analogous variants of both illusion figures or whether the claim should be generalized to the illusions produced by the classical figures, as well.

To the extent that the same visual mechanism is involved in the production of the classical Ponzo and Muller-Lyer illusions, it can be argued that corresponding alterations in stimulus variables should affect the magnitude of both illusions similarly. Since the claim of commonality of these two illusions rests on the assumption that the wedge of the Ponzo figure and the fins of the arrowfeather Muller-Lyer figure function identically, manipulations of the stimulus properties of these portions of the illusion figures are particularly pertinent. Using arrowfeather Muller-Lyer figures with fins approximately $25 \%$ the length of the center line, Weintraub, Tong, and Smith (1973) and Wickelgren (1965) have shown that increasing the lightness contrast of the fins relative to the center line increases the overestimation of center line length. Also, for the arrowfeather Muller-Lyer figure, it has been found that progressive increases in the length of the fins are accompanied first by increases, then by decreases, in the overestimation of the center line (Jaeger, 1975; Lewis, 1909). Thus, assuming analogous effects to exist for the Ponzo illusions, it can be predicted that increasing the lightness contrast of the wedge relative to the enclosed line should increase the overestimation of the enclosed line. Also, it can be expected that progressive increases in the length of the Ponzo wedge lines should be accompanied first by increases, then decreases, in the overestimation of the enclosed line. By testing these hypotheses, the present experiment will provide a 
further examination of the similarities between the Ponzo and Muller-Lyer illusions, as well as provide new data that may be beneficial in evaluating current explanations of the misestimations of line length produced by the Ponzo figure.

\section{METHOD}

\section{Subjects}

The subjects were 18 male and 18 female undergraduates ranging in age from 18 to 25 years. Each had visual acuities, corrected or uncorrected, of 20/20 or better, as measured by a Master orthorater.

\section{Stimuli}

Ponzo figures were constructed with black ( $3 \%$ reflectance) and light gray (67\% reflectance) Chartpak tape $1 \mathrm{~mm}$ in width. The illusion stimulus consisted of a wedge forming a 60 -deg angle that enclosed a single test line. The length of the sides of the wedge were 14,27 , or $40 \mathrm{~mm}$, and the single enclosed line was always $11 \mathrm{~mm}$ in length. To create the four contrast conditions, the Ponzo figures were constructed so as to have a black wedge and a gray enclosed line (BG), a black wedge and enclosed line (BB), a gray wedge and enclosed line (GG), or a gray wedge and black enclosed line (GB). These figures were mounted on an unlined white file card with the apex of the wedge $37 \mathrm{~mm}$ to the left of a central fixation point. The enclosed line appeared $26 \mathrm{~mm}$ to the left of the central fixation point, and a comparison line, whose contrast always matched that of the enclosed line, was mounted $26 \mathrm{~mm}$ to the right of the fixation point. The length of the comparison line ranged from 7 to $15 \mathrm{~mm}$ in $1-\mathrm{mm}$ steps. The width of the entire stimulus array was $3 \mathrm{deg} 2 \mathrm{~min}$ $40 \mathrm{sec}$, and its maximum height was $1 \mathrm{deg} 50 \mathrm{~min} 50 \mathrm{sec}$.

In addition to the illusion stimuli, control stimuli were constructed consisting of a single black test line $11 \mathrm{~mm}$ in length that was centered $26 \mathrm{~mm}$ to the left of a central fixation point. A black comparison line, which varied in length from 8 to $14 \mathrm{~mm}$ in $1-\mathrm{mm}$ steps, was centered $26 \mathrm{~mm}$ to the right of the fixation point. The width of this stimulus array was 2 deg $24 \mathrm{~min} 2 \mathrm{sec}$, and the maximum height was $38 \mathrm{~min} 36 \mathrm{sec}$.

\section{Procedure}

The subjects were randomly assigned to one of the three experimental groups with the stipulation that an equal number of males and females be represented in each group. Group 1 saw the Ponzo figure with $14-\mathrm{mm}$ wedge lines; Group 2 saw the Ponzo figure with 27-mm wedge lines; and Group 3 saw the Ponzo figure with 40-mm wedge lines.

Five minutes before the beginning of each testing session, subjects began a period of dark adaptation. During dark adaptation, instructions were given. The subjects were told that for each stimulus presented they should respond "longer" if the comparison line appeared longer than the enclosed line of the Ponzo figure and "shorter" if the comparison line appeared shorter than the enclosed line. No "equal to" responses were accepted.

Stimuli were presented for $700 \mathrm{msec}$ in a three-channel tachistoscope (Scientific Prototype, Model GB), with level of illumination at the eyepiece set at $16.5 \mathrm{~mL}$. Each experimental session began and ended with a control condition, in which subjects judged the length of the enclosed line in the Ponzo figure. Both control conditions contained four trials, each trial consisting of seven judgments. The order of stimulus presentation was determined by a converging method of limits (Clem \& Pollack, 1975), and initiation of each trial with either the longest or the shortest comparison line was counterbalanced following an ABBA design.

After the control trials, the subjects immediately began the experimental task of judging the relative lengths of the enclosed and comparison lines. Each subject saw only one of the three wedge conditions but saw all four levels of the lightness contrast variable. For each of these contrast levels, eight converging method-of-limits trials were given, with 11 judgments/trial. Again, initiation of each trial with either the longest or shortest comparison line was counterbalanced following an ABBA design, and the order of presentation of the contrast conditions for each subject was determined in accordance with a balanced-square design.

\section{RESULTS}

Points of subjective equality (PSEs), defined as the point at which a changed response was maintained for three consecutive judgments, were calculated for each subject on each trial. These scores were converted to illusion magnitude scores by subtracting the mean PSE of the control trials from the mean PSE of the trials in each experimental condition for each subject. Averaging these illusion magnitude scores across subjects produced the data in Figure 1. Criterion $t$ tests revealed that significant overestimation of the enclosed line was obtained for the BG figure with short wedge lines, for the $\mathrm{BG}, \mathrm{BB}$, and $\mathrm{GG}$ figures with intermediate wedge lines, and for the $\mathrm{BG}, \mathrm{BB}$, and $\mathrm{GB}$ figures with long wedge lines. None of the other departures from zero illusion was significant.

The illusion magnitude scores of each subject were used to perform a two-way mixed-model analysis of variance. Significant main effects were obtained for the wedge length $[\mathrm{F}(2,33)=3.834, \mathrm{p}<.01]$ and lightness contrast $[\mathrm{F}(3,99)=28.006, \mathrm{p}<.001]$ conditions. Also, the Wedge Length by Lightness Contrast interaction was significant $[F(6,99)=7.272, p<.001]$. Inspection of Figure 1 suggests that this interaction results from the

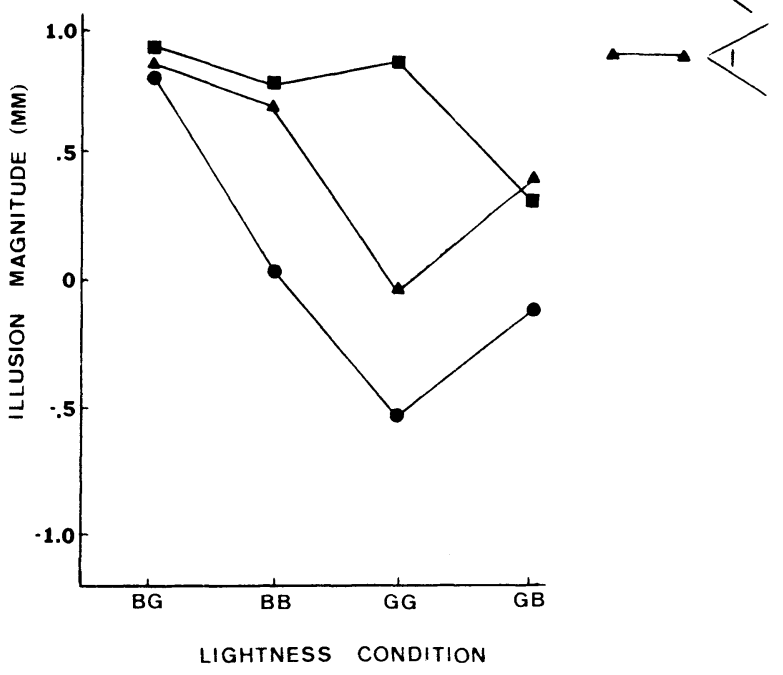

Figure 1. Illusion magnitude as a function of lightness contrast with wedge line length as parameter. Each data point is obtained by averaging across 12 subjects, with eight converging method-of-limits trials per subject. Positive scores represent overestimation of the enclosed line; negative scores represent underestimation. 
fact that manipulations of lightness contrast produce a pattern of illusion magnitude scores for the figure with intermediate wedge lines that differs from the patterns of scores produced by the figures with short and long wedge lines. This analysis is confirmed by three tests of trend that indicate that, for the figure with intermediate wedge lines, only the linear component of the function relating lightness contrast and illusion magnitude was significant, but for the figures with short and long wedge lines, both linear and quadratic components were significant.

To complete the data analysis, Newman-Keuls tests were employed to perform paired comparisons between different lightness contrast variations of Ponzo figures having matching wedge lines. For the figures with the shortest wedge lines, the BG vs. GG, BG vs. GB, BG vs. $\mathrm{BB}, \mathrm{BB}$ vs. $\mathrm{GG}$, and $\mathrm{GG}$ vs. $\mathrm{GB}$ pairings all yielded significant differences. For the figures having intermediate wedge lines, significant differences were obtained for the $\mathrm{BG}$ vs. $\mathrm{GB}, \mathrm{BB}$ vs. GB, and GG vs. GB pairings. Finally, for the figures with long wedge lines, the $B G$ vs. $\mathrm{GG}, \mathrm{BG}$ vs. $\mathrm{GB}, \mathrm{BB}$ vs. $\mathrm{GG}$, and $\mathrm{GG}$ vs. GB pairings produced significant differences.

\section{DISCUSSION}

The data obtained here clearly demonstrate that lightness contrast and wedge line length are potent variables affecting the magnitude of the Ponzo illusion. For the Ponzo figure with intermediate wedge lines, it was found that increases in the lightness contrast of the wedge relative to the enclosed line produced progressive increases in the overestimation of enclosed line length. For the figures with short and long wedge lines, a similar trend emerged, except that in the GG contrast conditions the apparent length of the enclosed line was less than that in the GB conditions. It should be noted that the lightness contrast effect obtained here with the Ponzo figure having intermediate wedge lines corresponds exactly to Wickelgren's (1965) report of the effect of lightness contrast on the arrowfeather MullerLyer illusion. As previously indicated, she found that, for this illusion figure, increases in the contrast of the fins relative to the center line produced progressive increases in the overestimation of the center line. There is also at least partial comparability between Wickelgren's (1965) data and those reflecting the effects of lightness contrast on the short and long wedged Ponzo figures, since for these figures, the overestimation of the enclosed line in the BG contrast conditions is consistently larger than that in the BB, GG, and GB conditions.

Turning now to the wedge length variable, it was found that increases in the length of the sides of the Ponzo wedge were accompanied by increases in the overestimation of the enclosed line. This overestimation was maximal for the figure with intermediate wedge lines, thus showing that, for the Ponzo illusion, apparent enclosed line length is a nonmonotonic function of the length of the wedge lines. Here, it should be recalled that both Jaeger (1975) and Lewis (1909) reported a similar nonmonotonic effect of fin length for the Muller-Lyer illusion. Using the arrowfeather figure, these investigators demonstrated that progressive increases in the length of the fins produced, first, increases, then decreases, in the overestimation of the center line. The similarity in these nonmonotonic relationships together with the demonstration of analogous lightness contrast effects for the Ponzo and Muller-Lyer illusions substantiates Fisher's (1967) and Pressey's (1971) claim that these two illusions are products of the same perceptual process. More specifically, it appears that the portion of the Ponzo wedge that extends outward from the enclosed line activates the same length-distorting visual mechanism as do the fins of the arrowfeather Muller-Lyer figure.

To close, it may be useful to consider the implications of these data for current theoretical accounts of the Ponzo illusion. ${ }^{1}$ After noting the structural similarities of the Ponzo and Muller-Lyer figures, both Fisher $(1967,1968,1969,1973)$ and Pressey (1971; Pressey, Butchard, \& Scrivner, 1971) presented explanations of these line length distortions. Pressey suggested that judgments of the length of the enclosed line are assimilated to the length of the longer extents that run parallel to the enclosed line and have endpoints defined by the wedge of the Ponzo figure. However, the finding of an optimal wedge line length seems inconsistent with assimilation theory because, as the length of the wedge lines is progressively increased, longer extents are provided to which the judged length of the enclosed line should be assimilated. These longer extents appear progressively closer to the fixation point at the center of the stimulus array and so are certainly well within the subject's attentive field. Moreover, the finding that manipulation of the contrast of the contours of some Ponzo figures can eliminate the overestimation of the enclosed line is contradictory to an assimilation hypothesis, since the extents to which the enclosed line are to be assimilated remain unaltered by contrast variations.

The effects of the lightness contrast variable noted above are more in line with Fisher's $(1967,1968,1969,1973)$ contention that the Ponzo illusion must be at least partially attributable to a contour interaction process. Although Fisher has not yet specified the mechanism mediating contour interactions, he has claimed that the Ponzo illusion is a manifestation of the fact that contours enclosed by a wedge will be progressively increased in length as their proximity to the wedge increases. As Fisher $(1968,1973)$ has shown, this distortion gradient principle is applicable when multiple lines are enclosed by a Ponzo wedge, but the data obtained here show that lengthening of the wedge line significantly affects illusion magnitude despite the constant proximity of the wedge and enclosed lines. Thus, while it appears that Fisher's distortion gradient principle cannot offer an explanation for all the vagaries of the Ponzo illusion, it does at least seem evident that a satisfactory account of this length distortion will need to incorporate a contour interaction process. The latter conclusion is supported by Wagner's (1977) ontogenetic study of the outline Ponzo figure, which reveals a decline in illusion susceptibility with increasing chronological age. Wagner, as well as Quina and Pollack (1972), contends that this Type 1 age trend is the result of physiological aging in the visual system that decreases sensitivity to contour and consequently weakens any illusion dependent for its existence upon contour interactions.

\section{REFERENCES}

Clem, R. K., \& Pollack, R. H. Illusion magnitude as a function of visual field exposure. Perception \& Psychophysics, 1975, $17,450-454$

DAY, R. H., \& Dickinson, R. G. Apparent length of the arms of acute and obtuse angles, and the components of the MullerLyer illusion. Australian Journal of Psychology, 1976, 28, 137-148.

Fisher, G. H. A common principle relating to the MullerLyer and Ponzo illusions. American Journal of Psychology, 1967, 80, 626-631.

Fisher, G. H. Gradients of distortion seen in the context of the Ponzo illusion and other contours. Quarterly Journal of Experimental Psychology, 1968, 20, 212-217.

Fisher, G. H. Towards a new explanation for the geometrical illusions. I. The properties of contours which induce illusory distortion. British Journal of Psychology, 1969, 60, 179-185.

Fisher, G. H. Towards a new explanation for the geometrical illusions. II. Apparent depth or contour proximity? British Journal of Psychology, 1973, 64, 607-621. 
Gregory, R. L. Distortion of visual space as inappropriate constancy scaling. Nature, 1963, 199, 678-680.

Gregory, R. L. Comments on L. E. Krueger's "disconfirming evidence" of R. L. Gregory's theory of illusions. Psychological Review, 1972, 79, 540-541.

JAEGER, T. Effect of changes in fin-length on apparent shaftlength and depth in the Muller-Lyer illusion. Perceptual and Motor Skills, 1975, 41, 79-84.

KRUEGER, L. E. Gregory's theory of illusions: Some disconfirming evidence in the case of the Muller-Lyer illusion. Psychological Review, 1972, 79, 538-539.

LEwis, E. O. Confluxion and contrast effects in the MullerLyer illusion. British Journal of Psychology, 1909, 3, 21-41.

Pressey, A. W. An extension of assimilation theory to illusions of size, area, and direction. Perception \& Psychophysics, 1971, 9, 172-176.

Pressey, A. W., Butchard, N., \& Scrivner, L. Assimilation theory and the Ponzo illusion: Quantitative predictions. Canadian Journal of Psychology, 1971, 25, 486-497.

Quina, K., \& Pollack, R. H. Parametric investigation of the Ponzo illusion under conditions of tachistoscopic exposure. Proceedings of the 79th Annual Convention of the American Psychological Association, 1971, 6, 77-78.

Quina, K., \& Pollack, R. H. Effects of test line position and age on the magnitude of the Ponzo illusion. Perception \& Psychophysics, 1972, 12, 253-256.

Wagner, D. A. Ontogeny of the Ponzo illusion: Effects of age, schooling, and environment. International Journal of Psychology, 1977, 12, 161-176.
Warren, R. M., \& Bashrord, J. A. Muller-Lyer illusions: Their origin in processes facilitating object recognition. Perception. 1977, 6, 615-626.

Weintraub, D. J., Tong, L., \& Smith, A. J. Muller-Lyer versus size/reflectance illusion: Is the age-related decrement caused by a declining sensitivity to brightness contours? Developmental Psychology, 1973, 8, 6-15.

Wickelgren, B. G. Brightness contrast and length perception in the Muller-Lyer illusion. Vision Research, 1965, 5, 141-150.

\section{NOTE}

1. A critique of Gregory's (1963) constancy scaling explanation of the Ponzo illusion might be attempted on the grounds that progressive increases in the length of the wedge lines should provide stronger depth cues and therefore consistent increases in the apparent length of the enclosed line. In principle, this argument would parallel Krueger's (1972) contention that progressive increases in fin length of the arrowfeather Muller-Lyer should provide stronger depth cues and therefore consistent increases in the apparent length of the center line. However, Gregory (1972) and the present authors believe that such a criticism rests on a confusion of the roles of primary and secondary constancy scaling.

(Received for publication October 29, 1979.) 